\title{
Development of nanocrystalline laser ablated thick film array gas sensor
}

\begin{abstract}
A multi-layer thick film array sensor for gas sensing application including heater element, insulator layer, and interdigitated electrodes was designed and fabricated on alumina substrate. Tin dioxide and platinum nanopowder were used in the pellet form as the active and catalyst layers, respectively. Pulse laser ablation deposition (PLAD) technique was used to deposit the sensitive layer onto the electrode part of each sensor. Microstructural and morphological properties of the sensor surface were determined. Sensors were exposed to wood smoke and their sensitivity were measured and compared with the results of the sensors without catalyst layer.
\end{abstract}

Keyword: Array gas sensor; SnO2; Thick film; Laser ablation 